\section{Just under $1 \%$ of adults living in the community in England are estimated to have autism spectrum disorders}

\section{QUESTION}

Question: What is the prevalence of autism spectrum disorders (ASD) in adults in England, and what are the characteristics of adults with ASD?

Population: 7461 adults (age 16 years or older) living in the community. Participants were members of private households sampled for the third national Adult Psychiatric Morbidity Survey in England. To select households for participation, post code sectors were first stratified by socioeconomic status, then further stratified based on proportions of adults in non-manual labour jobs and proportion of households without a motor vehicle as reported in 2001 census data. Postal code sectors were sampled from each stratum, yielding 519 selected postal code sectors. From each of these selected sectors, 28 addresses were randomly selected. Households with at least one person older than 16 years were eligible. Of these 14532 addresses, 13171 met eligibility criteria. Of the eligible households, 7461 agreed to participate (57\% response rate). Survey data were weighted such that the results were representative of age, sex, region and area characteristics of the household population 16 years or older in England.

Setting: General community dwelling population, England; 2007.

Assessment: A three-phase assessment process was then used to identify individuals with ASD. A subset of questions from the validated Autism Spectrum Quotient-20 self-completion questionnaire was used in a phase 1 screen. Based on the results of this screen, a subset of participants was selected for phase 2 assessment (higher scores increased likelihood of being selected). Phase 2 assessments were based on the Autism Diagnostic Observation Schedule - Module 4 (ADOS-4). Participants with an ADOS-4 score of 10 or higher were considered to have ASD. To validate this threshold, a subset of participants, half of whom were thought to have ASD based on ADOS-4 score, were then included in a phase 3 developmental interview with family members or other suitable informants using the Autism Diagnostic Interview - Revised and Diagnostic Interview for Social and Communication Disorders. The three-phase screening resulted in 7461 phase 1 interviews
(7403 direct and 58 proxy interviews), 5102 of which met eligibility criteria for phase 2 , and of these 849 were selected, and 630 phase 2 interviews were conducted $(74.2 \%$ response rate). Of these, 60 were selected for phase 3 interviews, with 54 phase 3 interviews conducted.

Outcomes: Diagnosis of ASD, based on a score of $\geq 10$ on the ADOS-4.

\section{METHODS}

Design: Cross-sectional study.

\section{MAIN RESULTS}

Overall 19 individuals were identified as having ASD, as defined by an ADOS-4 threshold of 10 or greater. None of the identified cases had been previously diagnosed. The weighted prevalence of ASD in the English population 16 years or older was estimated as 9.8 per 1000 (95\% CI 3.0 to 16.5). The prevalence of ASD showed a gradual decline with increasing age, but this decrease was not statistically significant. Multivariable models found that ASD was significantly more common in men than women (OR 8.62, 95\% CI 2.2 to $34.5, p=0.002)$. ASD was less common with increasing educational level (OR of ASD with a unit increase in educational level $0.45,95 \%$ CI 0.21 to $0.99, p=0.049)$. ASD was significantly more common among people living in social housing than those living in a self- or family-owned home (OR 6.1, $95 \%$ CI 1.7 to $21.7, \mathrm{p}=0.03$ ).

\section{CONCLUSIONS}

In 2007, ASD was present in approximately $1 \%$ of the adult population in England. Prevalence was highest in men, those with low-education attainment and those in social housing. This is similar to the prevalence of ASD reported in recent studies of children up to the age of 15 , suggesting that the causes of autism are temporally constant.

\section{ABSTRACTED FROM \\ Brugha TS, McManus S, Bankart J, et al. Epidemiology of autism spectrum disorders in adults in the community in England. Arch Gen Psychiatry 2011;68:459-65.}

Correspondence to Traolach S Brugha, Department of Health Sciences, University of Leicester, Leicester General Hospital, Gwendolen Road, Leicester LE5 4PW, England, UK; tsb@le.ac.uk

Sources of funding National Health Service Information Centre for Health and Social Care, Department of Health, National Institute for Health Research and Department of Health.

- Reference are available only at http://ebmh.bmj.com
$\mathrm{B}$ rugha and colleagues present the prevalence of autism spectrum disorder (ASD) in adults using a stratified, multiphase random sample survey design from the third national survey of psychiatric morbidity in England. The authors hypothesised the rate of ASDs in early adulthood would be similar to that reported in older children and conclude that the prevalence of ASDs (9.8 per 1000) in adults is similar to recent estimates found in children in the UK. They suggest that this is evidence that ASD prevalence is temporally constant. This study is the first to show the empirical evidence to refute the notion that $A S D$ rates are increasing by associating ASD prevalence and age. It also highlights both the feasibility and importance of studying adult $A S D$ prevalence.
Measuring ASD prevalence within any age group is a complex endeavour. The authors are to be commended for demonstrating that an adult ASD epidemiological study is possible and for acknowledging the challenges faced including a small number of total cases identified ( $n=19)$, exclusion of adults residing in institutions and adults with severe intellectual disability and low phase 1 response rates (57\%). Interpreting their findings, particularly similarities in ASD prevalence in adults to younger birth cohorts, however, remains unsettled. Two recent ASD epidemiological studies in children, for example, suggest that ASD prevalence is at least $0.33^{1}-1.69^{2}$ times higher than those reported by Brugha and colleagues. Study differences such as variation in the methods used for case identification, prevalence estimates based on a weighted number of cases with wide $\mathrm{Cl}$ and incomplete ascertainment due to non-participation make cross study comparisons difficult. Hopefully, the present study represents one of many to explore the epidemiology of ASDs in adults as such studies in children demonstrate how widely prevalence estimates can vary based on methodology, population size and other factors. In summary, more research is needed to support or refute the premise that there has not been some real increase in autism risk over the last three decades.

\section{Judith Pinborough-Zimmerman, \\ Amanda V Bakian}

Department of Psychiatry, University of Utah, Salt Lake City, Utah, USA

Competing interests None. 